\title{
Forced patterns near a Turing-Hopf bifurcation
}

\author{
Chad M. Topaz \\ Department of Mathematics, Statistics, and Computer Science, Macalester College, St. Paul, Minnesota 55105, USA
}

Anne J. Catllá

Department of Mathematics, Wofford College, Spartanburg, South Carolina 29303, USA

(Received 2 July 2009; revised manuscript received 29 December 2009; published 26 February 2010)

\begin{abstract}
We study time-periodic forcing of spatially extended patterns near a Turing-Hopf bifurcation point. A symmetry-based normal form analysis yields several predictions, including that (i) weak forcing near the intrinsic Hopf frequency enhances or suppresses the Turing amplitude by an amount that scales quadratically with the forcing strength, and (ii) the strongest effect is seen for forcing that is detuned from the Hopf frequency. To apply our results to specific models, we perform a perturbation analysis on general twocomponent reaction-diffusion systems, which reveals whether the forcing suppresses or enhances the spatial pattern. For the suppressing case, our results are consistent with features of previous experiments on the chlorine dioxide-iodine-malonic acid chemical reaction. However, we also find examples of the enhancing case, which has not yet been observed in experiment. Numerical simulations verify the predicted dependence on the forcing parameters.
\end{abstract}

DOI: 10.1103/PhysRevE.81.026213

PACS number(s): 05.45.-a, 47.54.- r, 82.40.Ck

\section{INTRODUCTION}

Turing patterns, originally conjectured as the basis for biological morphogenesis [1], arise in such diverse fields as ecology, materials science, and astrophysics [2-4]. Originating through a symmetry-breaking bifurcation of a homogenous steady state destabilized by diffusion, Turing patterns were first produced in experiment in the chlorite-iodidemalonic acid (CIMA) chemical reaction [5]. The CIMA reaction and its close variant, the chlorine dioxide-iodinemalonic acid (CDIMA) reaction, have rich pattern forming behavior, producing spots, stripes, rhombs, hexagons, and superlattices [6-9].

Many systems that form Turing-type patterns can also display a Hopf bifurcation to time-periodic solutions, and the interaction of these instabilities has been implicated in a variety of natural phenomena. Within the realm of biological and physical models, these phenomena include: chaos in predator-prey systems [10]; slow oscillations of coherent brain activity in the cerebral cortex [11]; propagating and standing fronts in arrays of resistively coupled nonlinear LC oscillators [12]; vortex glasses, chaos, localized structures, and mosaic patterns in optical parametric oscillators [13]; and various complex spatiotemporal behaviors in semiconductor charge transport $[14,15]$.

However, the Turing-Hopf interaction has arguably received the most focus vis-a-vis chemical experiments. Chemical phenomena attributed to the interaction of Turing and Hopf modes include: one-dimensional "spirals" in the CIMA reaction [16]; localized oscillations in the CDIMA reaction [17]; attenuation of oscillations and increased spatial coherence in the CDIMA reaction with random external spatiotemporal forcing $[18,19]$; and oscillatory Turing patterns, oscillons, and jumping and bubble waves in the BelousovZhabotisnky aerosol OT (BZ-AOT) reaction [20-22]. Foundational theoretical investigations [23-25] have studied the interaction both in canonical chemical models such as the Lengyel-Epstein model [26] and the Brusselator [27], and from a model-independent, symmetry-based perspective.

The discovery that the CDIMA reaction is photosensitive [28] has allowed an investigation of Turing pattern control. Spatial, temporal, and spatiotemporal forcing have been shown to induce a transition between patterns $[29,30]$, to introduce new localized [31] and complex [32] patterns, or simply to suppress patterns [33]. Turing systems that both contain a Hopf instability and are susceptible to external forcing pose an intriguing challenge. To control the system in a prescribed way, one must understand the delicate interplay of the two instabilities with the forcing.

In this paper, we study time-periodic forcing of spatially extended systems near a codimension-two Turing-Hopf bifurcation point. We give special attention to the forcing's effect on the Turing pattern. The forcing drives the Hopf mode, which in turn enhances or suppresses the Turing pattern modes to which it is coupled. Our results are threefold. First, in Sec. II we study weakly forced Turing-Hopf bifurcations from a symmetry-based (model-independent) perspective. Our results augment the existing theoretical literature on the Turing-Hopf normal form [23-25]. In the forced normal form, of special interest is the coefficient of the term that couples the Hopf mode to the Turing mode, whose sign dictates whether the forcing enhances or suppresses Turing patterns. We predict the dependence of the solutions on forcing amplitude and frequency and, as an example, relate our results to an experiment on the forced CDIMA system. Then, in Sec. III, we begin with a generic two-component reactiondiffusion system and use perturbation theory to calculate normal form coefficients. The result of the calculation applies to specific two-component systems such as the Lengyel-Epstein model, the Brusselator, and so forth and determines, as a function of the reaction kinetics, the qualitative effect of the forcing. Though previous work on the CDIMA reaction and Lengyel-Epstein model found only the suppressing effect $[33,34]$, we find enhancement in other models. Finally, in Sec. IV we verify some of our symmetry predictions via numerical simulations. 


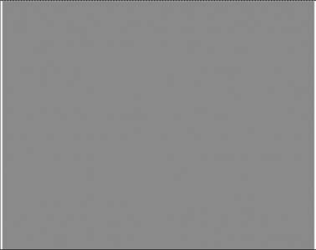

(a)

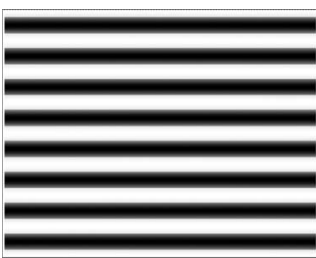

(e)

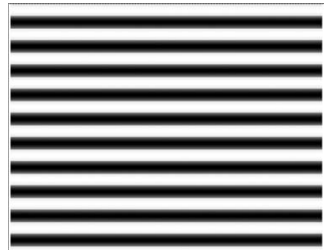

(b)

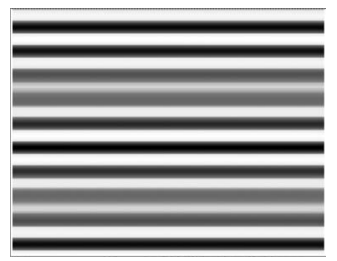

(f)

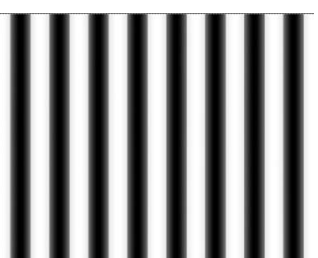

(c)

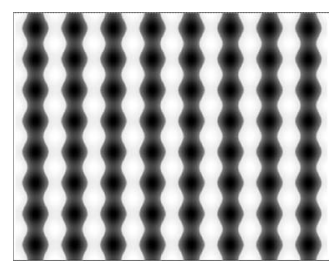

(g)

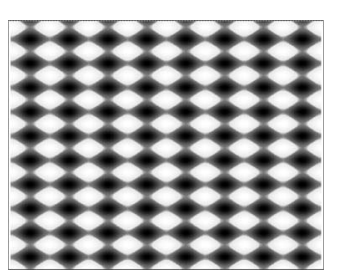

(d)

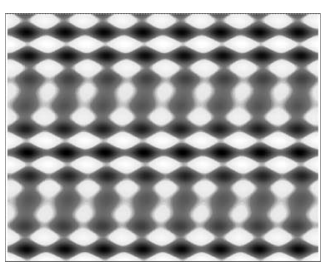

(h)

FIG. 1. Schematics in space (horizontal) time (vertical) of solutions to forced Turing-Hopf normal form (2). Shading corresponds to the underlying physical field $u$, e.g., the leading terms in Eq. (1). (a)-(d) The (well-known) solutions for the unforced case, namely trivial, Hopf, Turing, and mixed mode. (e)-(h) Corresponding leading-order perturbed solutions for weak forcing; see Sec. II.

\section{SYMMETRY ANALYSIS}

Consider a spatially extended system with a spatially homogeneous base state (zero, without loss of generality) and a codimension-two point in parameter space where steady, spatially periodic (Turing) modes and a spatially homogenous, time-periodic (Hopf) mode bifurcate simultaneously. For expository purposes, assume a simple stripe pattern. Close to the bifurcation point, let

$$
\mathbf{u}=z_{T}\left(t_{2}\right) \mathbf{v}_{T} e^{i \mathbf{q} \cdot \mathbf{x}}+z_{H}\left(t_{2}\right) \mathbf{v}_{H} e^{i \omega_{H} t}+\text { c.c. }+\cdots,
$$

where $\mathbf{u}$ describes the system state (e.g., chemical concentrations), $z_{T, H}\left(t_{2}\right)$ are the slowly varying amplitudes of the Turing and Hopf modes $\mathbf{v}_{T, H}, t_{2}$ is a slow-time variable, $\mathbf{q}$ is the wave vector of the pattern with wave number $q_{c}=|\mathbf{q}|$ as determined by linear stability theory, $\omega_{H}$ is the Hopf frequency, and the dots represent damped modes. Assume time-periodic forcing $f(t)$ which we take without loss of generality to have zero mean. For sufficiently weak forcing, the only frequency component of $f(t)$ entering the weakly nonlinear description will be that closest to $\omega_{H}$. Call this frequency $\omega_{f}$ and its strength $f_{H}$. Define the detuning $\Delta=\omega_{f}-\omega_{H}$ and assume $\Delta=0$ for now; later, we relax this assumption. Note that our bifurcation scenario differs from that in [35] and related works, which examine Turing bifurcations of an underlying oscillatory state.

Equations for the slow-time evolution of $z_{T, H}$ must respect the spatial symmetries of the underlying system [36], namely, translation by $\Delta \mathbf{x}\left(z_{T} \rightarrow z_{T} e^{i \mathbf{q} \cdot \Delta \mathbf{x}}\right)$ and inversion through the origin $\left(z_{T} \rightarrow \bar{z}_{T}\right)$. Forcing breaks the temporal symmetry of the problem, but it may be recast as a parameter symmetry by incorporating the action of time translation by $\Delta t$ on the forcing function (see, e.g., $[37,38]$ ) so that the full symmetry is $\left(z_{H},\left|f_{H}\right|\right) \rightarrow\left(z_{H},\left|f_{H}\right|\right) e^{i \omega_{H} \Delta t}$. The cubic amplitude equations are

$$
\dot{z}_{T}=\lambda z_{T}+\operatorname{sign}\left(g_{1}\right)\left|z_{T}\right|^{2} z_{T}+g_{2}\left|z_{H}\right|^{2} z_{T}
$$

$$
\dot{z}_{H}=\left|f_{H}\right|+\mu z_{H}+g_{3}\left|z_{H}\right|^{2} z_{H}+g_{4}\left|z_{T}\right|^{2} z_{H} .
$$

Here, $\lambda, g_{1,2} \in \mathbb{R}$ and $\mu, g_{3,4} \in \mathrm{C}$. We have performed a phase shift and rescaling so that the forcing appears as $\left|f_{H}\right|$; we also scaled such that the self-interaction coefficient of $z_{T}$ is $\operatorname{sign}\left(g_{1}\right)= \pm 1$. Throughout the symmetry analysis, assume a supercritical bifurcation to stripes, and thus set $\operatorname{sign}\left(g_{1}\right)=-1$. Also assume that $\operatorname{Re}\left(g_{3}\right)<0$ for a supercritical Hopf bifurcation, so that the amplitude equations give a valid weakly nonlinear description. For weak forcing, terms smaller than $\mathcal{O}\left(f_{H}\right)$ have no effect at leading order and are ignored.

In the absence of forcing $\left(f_{H}=0\right)$ there are four solutions to Eq. (1): the trivial solution $\left(z_{T, H}=0\right)$, a pure Hopf mode $\left(z_{T}=0, z_{H} \neq 0\right)$, a pure Turing mode $\left(z_{T} \neq 0, z_{H}=0\right)$, and a mixed mode $\left(z_{T, H} \neq 0\right)$; see, e.g., [25]. Space-time plots of these solutions are shown in the top row of Fig. 1. To calculate how weak forcing perturbs these solutions, perform a regular perturbation expansion. Assume $\left|f_{H}\right|$ is $\mathcal{O}(\eta)$ where $\eta \ll 1$ is a book-keeping parameter. Let

$$
z_{T}=z_{T}^{(0)}+\eta z_{T}^{(1)}+\ldots, \quad z_{H}=z_{H}^{(0)}+\eta z_{H}^{(1)}+\ldots
$$

Substituting Eq. (3) into Eq. (2), collecting terms in $\eta$, and solving at each power leads to the following four perturbed solutions, where we have retained only the leading terms and reconstituted the expressions in $\eta$. We describe the solutions to the perturbed cases below and provide space-time plots of the solutions in the bottom row of Fig. 1.

(i) Perturbed trivial solution. Here $z_{T}=0, z_{H} \approx-\left|f_{H}\right| / \mu$. The physical solution (1) is an $\mathcal{O}\left(f_{H}\right)$ spatially homogenous oscillation with frequency $\omega_{H}$.

(ii) Perturbed Hopf mode. In this case, 


$$
\begin{gathered}
z_{T}=0, \quad z_{H} \approx R e^{i \Omega t}+\left|f_{H}\right|\left(A+B e^{2 i \Omega t}\right), \\
R\left(\mu, g_{3}\right)=\sqrt{-\operatorname{Re}(\mu) / \operatorname{Re}\left(g_{3}\right)}, \\
\Omega\left(\mu, g_{3}\right)=\operatorname{Im}(\mu)+\operatorname{Im}\left(g_{3}\right) R^{2} .
\end{gathered}
$$

$A$ and $B$ are $\mathcal{O}(1)$ constants depending on coefficients in Eq. (2); their exact expressions are not key to our discussion but may be obtained by substituting Eq. (4) into Eq. (2). The physical solution is an $\mathcal{O}(1)$ spatially homogeneous oscillation with frequency $\omega_{H}+\Omega$ superposed on $\mathcal{O}\left(f_{H}\right)$ oscillations with frequencies $\omega_{H}$ and $\omega_{H}+2 \Omega$. The $\mathcal{O}\left(f_{H}\right)$ correction is consistent with results from [39-41].

(iii) Perturbed Turing mode. We have

$$
\begin{gathered}
\left|z_{T}\right| \approx \sqrt{\lambda}\left(1+\frac{g_{2}\left|f_{H}\right|^{2}}{2 \lambda|\tilde{\mu}|^{2}}\right), \\
z_{H} \approx-\frac{\left|f_{H}\right|}{\tilde{\mu}}, \quad \tilde{\mu}=\mu+\lambda g_{4} .
\end{gathered}
$$

The physical solution is an $\mathcal{O}(1)$ spatial pattern superposed on an $\mathcal{O}\left(f_{H}\right)$ spatially homogenous oscillation.

(iv) Perturbed mixed mode. In this case, $z_{T}$ and $z_{H}$ are both time dependent,

$$
\begin{gathered}
\left|z_{T}\right| \approx \sqrt{\lambda+g_{2} \widetilde{R}^{2}}+\left|f_{H}\right| \widetilde{C} \cos (\tilde{\Omega} t+\phi), \\
z_{H} \approx \widetilde{R} e^{i \tilde{\Omega} t}+\left|f_{H}\right|\left(\widetilde{A}+\widetilde{B} e^{2 i \tilde{\Omega} t}\right),
\end{gathered}
$$

where $\tilde{R}=R\left(\tilde{\mu}, \widetilde{g}_{3}\right), \tilde{\Omega}=\Omega\left(\tilde{\mu}, \widetilde{g}_{3}\right)$, and $\tilde{g}_{3}=g_{3}+g_{2} g_{4}$. The $\mathcal{O}(1)$ constants $\widetilde{A}, \widetilde{B}, \widetilde{C}$, and $\phi$ are obtained by substitution. The physical solution is an $\mathcal{O}(1)$ spatial pattern with $\mathcal{O}\left(f_{H}\right)$ breathing at frequency $\widetilde{\Omega}$, superposed on oscillations similar to case (ii).

To explore mechanisms for suppressing and enhancing Turing patterns, we consider further case (iii). For comparison with experimental results, we now allow detuning from $\omega_{H}(\Delta \neq 0)$, in which case $(5)$ becomes

$$
\left|z_{T}\right| \approx \sqrt{\lambda}\left(1+\frac{g_{2}\left|f_{H}\right|^{2}}{2 \lambda|\tilde{\mu}-i \Delta|^{2}}\right), \quad z_{H} \approx-\frac{\left|f_{H}\right| e^{i \Delta t}}{\tilde{\mu}-i \Delta} .
$$

The forcing's effect hinges on the sign of $g_{2}$. If $g_{2}<0$ $\left(g_{2}>0\right)$ the forcing suppresses (enhances) the Turing pattern. If $g_{2}<0$, weak forcing reduces the pattern amplitude by a relative amount proportional to $\left|f_{H}\right|^{2}$. Furthermore, since $\left|z_{T}\right|>0$, it follows that the domain of pattern existence is shifted from $\lambda>0$ (unforced case) to $\lambda>-g_{2}\left|f_{H}\right|^{2}|\tilde{\mu}-i \Delta|^{-2}>0$. If $g_{2}>0$, the forcing enhances the pattern and shifts the bifurcation in the opposite direction, so that a pattern appears in the forced system for $\lambda$ values where it would not exist in the unforced system. By optimizing $\left|z_{T}\right|$ in Eq. (7) one sees the enhancement/suppression is strongest for $\Delta_{\text {opt }}=\operatorname{Im}(\tilde{\mu}) \neq 0$ and decays away from this maximum. Note that since $\tilde{\mu}$ depends linearly on $\mu$ and $\lambda$, the shift of $\Delta_{\text {opt }}$ away from zero is small if we are close to the codimension-two point. A straightforward stability calculation shows the rate of suppression (i.e., the eigenvalue of the perturbed Turing mode) is approximately $-g_{2}\left|f_{H}\right|{ }^{2} \lambda^{-1} \mid \tilde{\mu}$ $-\left.i \Delta\right|^{-2}$.

The results above are qualitatively consistent with experiments on the CDIMA chemical reaction [33] which considered time-periodic forcing applied to stable Turing patterns (though the experimental patterns were not necessarily close to onset). First, forcing was observed to suppress the pattern, corresponding to our case $g_{2}<0$. Second, suppression was strongest for $\omega_{f} \approx \omega_{H}$, in agreement with our explanation of coupling to the Hopf mode as the (indirect) control mechanism. Third, the rate of pattern suppression has a maximum near $\Delta=0$. Finally, the numerical simulations of [33], taken as a qualitative model of the experiment, showed that the domain of Turing pattern existence was shifted, with a critical curve in the $\left(\left|f_{H}\right|, \Delta\right)$ plane having a minimum near $\Delta=0$, which follows directly from our expression for shifted domain existence.

\section{PERTURBATION ANALYSIS}

To connect the symmetry results to models, consider a general reaction-diffusion system for species $u(\mathbf{x}, t)$ and $v(\mathbf{x}, t)$ which in the absence of forcing has a spatially homogenous state assumed without loss of generality to be $u=v=0$. Similar to [42], write the governing equations as

$$
\begin{aligned}
\partial_{t}\left(\begin{array}{l}
u \\
v
\end{array}\right) & =\mathbf{L}\left(\begin{array}{l}
u \\
v
\end{array}\right)+\mathbf{R}(u, v)+\mathbf{f}(t), \\
\mathbf{L} & \equiv\left(\begin{array}{cc}
a+\nabla^{2} & b \\
c & d+K \nabla^{2}
\end{array}\right),
\end{aligned}
$$

where $\mathbf{L}$ is the linear operator, $\mathbf{f}(t)$ is spatially homogeneous periodic forcing with zero mean, and $\mathbf{R}$ contains nonlinear reaction terms and satisfies $\mathbf{R}(0,0)=\mathbf{0}$.

We first locate the codimension-two Turing-Hopf point via linear stability analysis. Consider the usual Fourier-type perturbations

$$
\mathbf{u}=\left(\begin{array}{l}
u \\
v
\end{array}\right) e^{\sigma t+i \mathbf{q} \cdot \mathbf{x}}
$$

where the perturbation has wave number $q=|\mathbf{q}|$ and growth rate $\sigma$. The eigenvalues $\sigma_{1,2}(q)$ of the matrix

$$
\mathbf{L}(q) \equiv\left(\begin{array}{cc}
a-q^{2} & b \\
c & d-K q^{2}
\end{array}\right),
$$

determine (in)stability. The conditions for the Hopf bifurcation are $\operatorname{Re} \sigma_{1,2}(0)=0, \operatorname{Im} \sigma_{1}(0)=-\operatorname{Im} \sigma_{2}(0) \neq 0$, which imply that $\operatorname{tr} L(0)=0$, det $L(0)>0$. Therefore, $d=-a$ and $a^{2}+b c<0$. The critical eigenvectors are $\mathbf{v}_{H}=\left(b,-a+i \omega_{H}\right)^{T}$ and its complex conjugate, where the Hopf frequency $\omega_{H}$ satisfies $\omega_{H}^{2}=-\left(a^{2}+b c\right)$. The conditions for a Turing bifurcation are $\operatorname{Re} \sigma_{1}\left(q_{c}\right)<\operatorname{Re} \sigma_{2}\left(q_{c}\right)=0$ where $q_{c} \neq 0$ is the critical wave number. This condition implies that $\operatorname{det} L\left(q_{c}\right)=0$. We also require all other modes to be damped at the bifurcation point, so that $\operatorname{Re} \sigma_{1,2}(q)<0$ for $q \neq 0, q_{c}$. This is guaranteed if $\operatorname{det} \mathbf{L}(q)$ has a minimum at $q_{c}$, from which it follows that $q_{c}^{2}=a(K-1) /(2 K)$. The critical eigenvector is $\mathbf{v}_{\mathbf{T}}=\left(-b, a-q_{c}^{2}\right)^{T}$. 

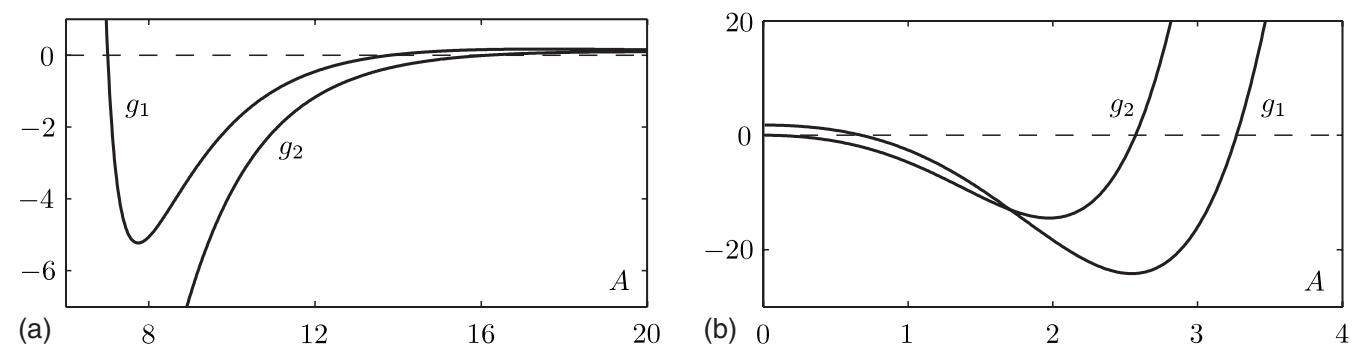

FIG. 2. Coefficients $g_{1,2}$ in Eq. (2) as computed from two reaction-diffusion models, namely, the Lengyel-Epstein model Eq. (14) and Brusselator Eq. (15). At the Turing-Hopf point, the coefficients in Eq. (2) depend only on the parameter $A$ in the governing equations. For each case, we focus on the interval of $A$ for which $g_{1}<0$ so that the bifurcation to a Turing pattern is supercritical. (a) In the Lengyel-Epstein equations, $g_{1}<0$ for $A \in[7.0,13.8]$. The coupling coefficient $g_{2}<0$, so that forcing suppresses the spatial pattern. (b) In the Brusselator, $g_{1}<0$ for $A \in[0.68,3.26]$. For $A \in[0.68,2.57], g_{2}<0$ so that forcing suppresses the spatial pattern. For $A \in(2.57,3.26], g_{2}>0$ so that forcing actually enhances the spatial pattern.

In summary, at the codimension-two point, the linear parameters in $a, b, c, d, K$ in Eq. (8a) must satisfy

$$
\begin{gathered}
d=-a, \\
a^{2}+b c<0, \\
a^{2}(K+1)^{2}+4 K b c=0, \\
b c<0 .
\end{gathered}
$$

The critical Turing and Hopf eigenvectors are

$$
\mathbf{v}_{T}=\left(\begin{array}{c}
-b \\
a-q_{c}^{2}
\end{array}\right), \quad \mathbf{v}_{H}=\left(\begin{array}{c}
a-q_{c}^{2} \\
-a+i \omega_{H}
\end{array}\right),
$$

and additionally

$$
\begin{gathered}
\left|\mathbf{q}_{c}\right|^{2}=q_{c}^{2}=\frac{a_{c}\left(K_{c}-1\right)}{2 K_{c}}, \\
\omega_{H}^{2}=-\left(a_{c}^{2}+b_{c} c_{c}\right)=K_{c} q_{c}^{4} .
\end{gathered}
$$

To compute the coefficients in Eq. (2), we perform a multiple time scales perturbation expansion for the case of weak forcing. The calculation, though straightforward, is algebraically tedious; we give some details in the Appendix.

As we are interested in the effect of forcing on Turing patterns, we focus on the sign of $g_{2}$, which we calculate for two different models. Consider first the Lengyel-Epstein model of the CDIMA reaction with forcing [26,33],

$$
\begin{gathered}
\dot{u}=A-u-4 u v\left(1+u^{2}\right)^{-1}-f(t)+\nabla^{2} u, \\
\dot{v}=\sigma\left[B\left\{u-u v\left(1+u^{2}\right)^{-1}+f(t)\right\}+D \nabla^{2} v\right],
\end{gathered}
$$

where $u$ and $v$ represent the reacting chemical species, $A, B$, and $\sigma$ are chemical parameters, and $D$ is a diffusion constant. Because there are three effective parameters affecting the linear problem, namely $A, \sigma B$, and $\sigma D$, and because there are two equalities [Eq. (11a) and (11c)] that the linear parameter must satisfy in order to be at the codimension-two point, all coefficients in Eq. (2) can be written as functions of $A$. Figure 2(a) shows $g_{1,2}(A)$. For all values of $A$ for which the Turing pattern bifurcates supercritically $\left(g_{1}<0\right)$, the coeffi- cient $g_{2}<0$, and thus forcing suppresses the pattern, in agreement with only suppression having been observed in CDIMA experiments. As a second example, consider the forced Brusselator [43],

$$
\begin{gathered}
\dot{u}=A-(B+1) u+u^{2} v+\nabla^{2} u+f(t), \\
\dot{v}=B u-u^{2} v+D \nabla^{2} v,
\end{gathered}
$$

for which all coefficients in Eq. (2) can again be written as functions of $A$. As shown in Fig. 2(b), when $g_{1}<0$, there is one subinterval in which $g_{2}>0$ so that forcing can enhance the spatial Turing pattern.

\section{NUMERICAL SIMULATIONS}

To verify the symmetry-based and perturbation results of Secs. II and III, we perform numerical simulations of the Brusselator Eq. (15). Simulations are carried out close to the codimension-two point in a one-dimensional periodic domain of size $16 \times 2 \pi / q_{c}$. We compute spatial derivatives pseudospectrally. The equations are evolved in real space using the Matlab package's built-in integrators (e.g., ODE45).

Figure 3 shows an example of Turing pattern enhance-

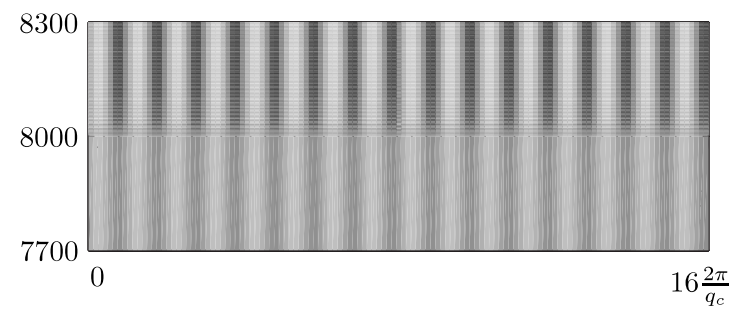

FIG. 3. Simulation of the Brusselator Eq. (15). Values of $u$ are indicated by shading in the $x-t$ plane. The simulation begins with $f(t)=0$ (no forcing). A random initial condition (not shown) evolves to a steady state Turing pattern by $t<8000$. At $t=8000$, we set $f(t)=0.01 \cos \left(\omega_{H} t\right)$, which enhances the Turing pattern (as seen in the sharper contrast between peaks and valleys). The chemical parameters are $A=3, B=9.998, D \approx 1.926$ for which $\lambda=-\operatorname{Re} \mu=0.001$ in Eq. (1). At the codimension-two point, $q_{c} \approx 1.47$ and $\omega_{H}=3$. 


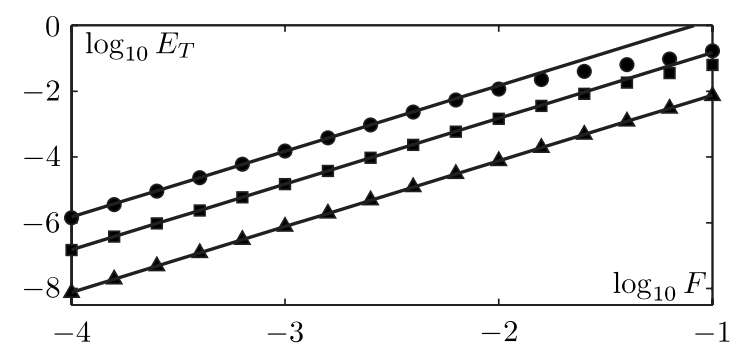

FIG. 4. Scaling of Turing pattern enhancement $E_{T}$ as a function of forcing strength for the Brusselator Eq. (15) with forcing $f(t)=F \cos \left(\omega_{H} t\right)$. Symbols correspond to simulations while the lines of slope 2 show the quadratic dependence on forcing strength predicted by Eq. (7). For all data, $A=3$ and hence the critical wave number $q_{c}$ and Hopf frequency $\omega_{H}$ are as in Fig. 3. For each data set, $\lambda=-\operatorname{Re} \mu$ in Eq. (2). () $B=9.98, D \approx 1.935$ for which

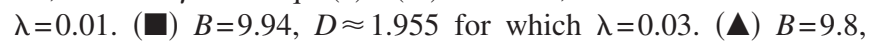
$D \approx 2.031$ for which $\lambda=0.1$.

ment. In this example, the chemical parameters are $A=3, B=9.998, D \approx 1.926$ for which $\lambda=-\operatorname{Re} \mu=0.001$ in Eq. (2)). At the codimension-two point, $q_{c} \approx 1.47$ and $\omega_{H}=3$. A random initial condition evolves to reach a steady Turing pattern. At time $t=8000$, we set $f(t)=0.01 \cos \left(\omega_{H} t\right)$. The resulting Turing pattern enhancement is visible as a sharper contrast between peaks and valleys.

In further simulations we quantify the Turing pattern enhancement $E_{T}$. To determine $E_{T}$, we allow the forced system to settle onto its attractor. We measure the time-averaged amplitude of the critical Turing mode, and from this subtract the corresponding value for the unforced case. Figure 4 shows $E_{T}$ as a function of forcing strength for simple harmonic forcing and for three different sets of chemical parameters. For each data set, $A=3$ in Eq. (15) but we vary $B$ and $D$ to achieve different distances from the codimension-two point, holding $\lambda=-\operatorname{Re} \mu$. The quadratic scaling of Eq. (7) holds over several decades, for sufficiently small forcing relative to the distance from the codimension-two point.

Figure 5(a) shows $E_{T}$ vs $\Delta$ for the same three sets of chemical parameters, with forcing strength $F$ fixed for each set. As predicted by Eq. (7), there exists an optimal detuning $\Delta_{\text {opt }}$ that maximizes $E_{T}$, and $\Delta_{\text {opt }} \rightarrow 0$ as the system parameters approach the codimension-two point. The inset, Fig. 5(b) verifies the functional dependence on $\Delta$ predicted by Eq. (7), namely, $E_{T}(\Delta)=c_{1} /\left[c_{2}+\left(\Delta-c_{3}\right)^{2}\right]$ for some constants $c_{1,2,3}$.

\section{CONCLUSION}

In this paper, we have studied the effect of time-periodic forcing on interacting Turing and Hopf instabilities. Our normal form results suggest a pattern control mechanism for spatially extended systems with these instabilities, including chemical reaction-diffusion systems. Although the symmetry analysis is performed for weak forcing and small-amplitude patterns, it nonetheless agrees with features of the experiments in [33]. Furthermore, we predict that the forcing may result in spatial pattern enhancement. Thus far, only suppression has been observed in experiment, but nonetheless, we

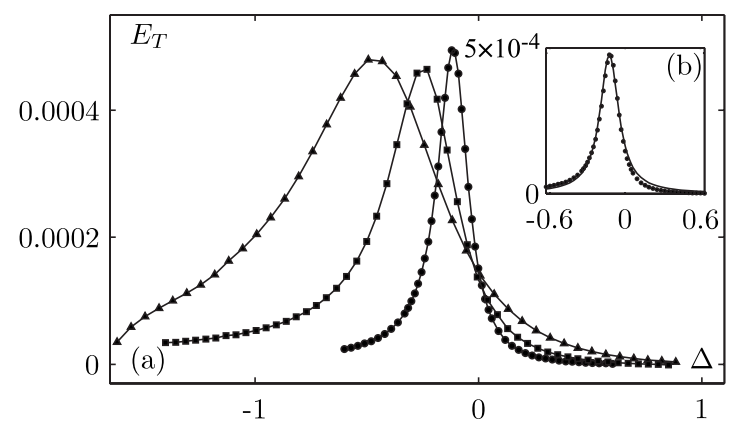

FIG. 5. Dependence of Turing pattern enhancement $E_{T}$ on frequency detuning $\Delta$ for Brusselator (15) with forcing $f(t)=F \cos \left[\left(\omega_{H}+\Delta\right) t\right]$. (a) The three sets of data correspond (same symbols) to the parameters in Fig. 4, except for the values of $F$. ( $F=0.001$. ( $\mathbf{\square}) F=0.003$. ( $\mathbf{\Delta}) F=0.01$. The lines guide the eyes. As predicted by Eq. (7), there is a $\Delta_{o p t}$ for which is effect is maximal and $\Delta_{\text {opt }} \rightarrow 0$ close to the codimension-two point. (b) Same data as in (a), but here the solid curve is a fit of the functional form $c_{1} /\left[c_{2}+\left(\Delta-c_{3}\right)^{2}\right]$ predicted by Eq. (7).

have demonstrated pattern enhancement with our perturbation analysis and numerical simulations. We hope that experimentalists might apply our results to look for the enhancing effect in other spatially extended systems with Turing and Hopf instabilities.

\section{ACKNOWLEDGMENTS}

C.M.T. was supported by NSF Grant No. DMS-0740484. A.J.C. was supported by NSF VIGRE Grant No. DMS9983320. We thank Andrew Bernoff, Jessica Conway, Milos Dolnik, Martin Golubitsky, Jeff Porter, David Schaeffer, and Mary Silber for helpful discussions.

\section{APPENDIX: PERTURBATION CALCULATION DETAILS}

Here we provide some details of the multiple-scales perturbation calculation of Sec. III that leads to expressions for the normal form coefficients in Eq. (2). Begin with the general two-component reaction-diffusion system Eq. (8a). For the weakly nonlinear analysis, it suffices to Taylor expand the reaction terms $\mathbf{R}$ to cubic order in $u, v$ which yields

$$
\begin{aligned}
& u_{t}=\nabla^{2} u+a u+b v+F_{2}(u, v)+F_{3}(u, v)+f_{1}(t), \\
& v_{t}=K \nabla^{2} v+c u+d v+G_{2}(u, v)+G_{3}(u, v)+f_{2}(t)
\end{aligned}
$$

$$
\begin{gathered}
F_{2}(u, v)=q_{11} u^{2}+q_{12} u v+q_{13} v^{2}, \\
G_{2}(u, v)=q_{21} u^{2}+q_{22} u v+q_{23} v^{2}, \\
F_{3}(u, v)=c_{11} u^{3}+c_{12} u^{2} v+c_{13} u v^{2}+c_{14} v^{3}, \\
G_{3}(u, v)=c_{21} u^{3}+c_{22} u^{2} v+c_{23} u v^{2}+c_{24} v^{3} .
\end{gathered}
$$

To study the slow-time evolution of the critical modes near the codimension-two point specified by Eq. (11), perform a two-timing perturbation expansion. Let 


$$
\begin{gathered}
\partial_{t} \rightarrow \partial_{t}+\epsilon^{2} \partial_{t_{2}}, \\
\{a, b, c, d, K\}=\{a, b, c, d, K\}_{c}+\epsilon^{2}\{a, b, c, d, K\}_{2}, \\
f_{1,2}(t) \rightarrow \epsilon^{3} f_{1,2}(t), \\
\mathbf{u}=\epsilon \mathbf{u}_{1}+\epsilon^{2} \mathbf{u}_{2}+\epsilon^{3} \mathbf{u}_{3},
\end{gathered}
$$

where $\epsilon \ll 1$ is a bookkeeping parameter, the subscript of $c$ indicates critical values as determined by Eq. (11), and $\mathbf{u}_{j}$ $=\left(u_{j}, v_{j}\right)^{T}$.

At $\mathcal{O}(\epsilon)$, the linear problem is recovered. The solution $\mathbf{u}_{1}$ is

$$
\left(\begin{array}{l}
u_{1} \\
v_{1}
\end{array}\right)=z_{T}\left(t_{2}\right)\left(\begin{array}{l}
u_{1 T} \\
v_{1 T}
\end{array}\right) e^{i q_{c} x}+z_{H}\left(t_{2}\right)\left(\begin{array}{l}
u_{1 H} \\
v_{1 H}
\end{array}\right) e^{i \omega_{H} t}+\text { c.c. },
$$

where $\mathbf{v}_{T}=\left(u_{1 T}, v_{1 T}\right)^{T}, \mathbf{v}_{H}=\left(u_{1 H}, v_{1 H}\right)^{T}$, and $q_{c}$ and $\omega_{H}$ are defined by Eqs. (12) and (13). Without loss of generality, we have assumed that the critical Turing wave vector is in the $x$ direction.

The $\mathcal{O}\left(\epsilon^{2}\right)$ problem is

$$
\partial_{t} \mathbf{u}_{2}=\mathbf{L}_{0} \mathbf{u}_{2}+\mathbf{N}_{2}\left(\mathbf{u}_{1}\right) \quad \text { where } \quad \mathbf{N}_{2}\left(\mathbf{u}_{1}\right)=\left(\begin{array}{c}
F_{2}\left(u_{1}, v_{1}\right) \\
G_{2}\left(u_{1}, v_{1}\right)
\end{array}\right) .
$$

The operator $\mathbf{L}_{0}$ is simply $\mathbf{L}\left(q_{c}\right)$ with $\mathbf{L}$ given by Eq. (10). Write the nonlinear terms as

$$
\mathbf{N}_{2}\left(\mathbf{u}_{1}\right)=\sum_{j=1}^{2} \mathbf{Q}_{2, j} z_{2, j}+\sum_{i=3}^{6}\left(\mathbf{Q}_{2, j} z_{2, j}+\text { c.c. }\right),
$$

where

$$
\mathbf{Q}_{2,1}=\left(\begin{array}{l}
2 q_{11} u_{1 T}^{2}+2 q_{12} u_{1 T} v_{1 T}+2 q_{13} v_{1 T}^{2} \\
2 q_{21} u_{1 T}^{2}+2 q_{22} u_{1 T} v_{1 T}+2 q_{23} v_{1 T}^{2}
\end{array}\right),
$$

$$
\mathbf{Q}_{2,2}=\left(\begin{array}{l}
2 q_{11}\left|u_{1 H}\right|^{2}+q_{12}\left(u_{1 H} \bar{v}_{1 H}+\bar{u}_{1 H} v_{1 H}\right)+2 q_{13}\left|v_{1 H}\right|^{2} \\
2 q_{21}\left|u_{1 H}\right|^{2}+q_{22}\left(u_{1 H} \bar{v}_{1 H}+\bar{u}_{1 H} v_{1 H}\right)+2 q_{23}\left|v_{1 H}\right|^{2}
\end{array}\right),
$$

$$
\mathbf{Q}_{2,3}=\left(\begin{array}{l}
2 q_{11} u_{1 T} u_{1 H}+q_{12}\left(u_{1 T} v_{1 H}+v_{1 T} u_{1 H}\right)+2 q_{13} v_{1 T} v_{1 H} \\
2 q_{21} u_{1 T} u_{1 H}+q_{22}\left(u_{1 T} v_{1 H}+v_{1 T} u_{1 H}\right)+2 q_{23} v_{1 T} v_{1 H}
\end{array}\right),
$$

$$
\mathbf{Q}_{2,4}=\left(\begin{array}{l}
2 q_{11} u_{1 T} \bar{u}_{1 H}+q_{12}\left(u_{1 T} \bar{v}_{1 H}+v_{1 T} \bar{u}_{1 H}\right)+2 q_{13} v_{1 T} \bar{v}_{1 H} \\
2 q_{21} u_{1 T} \bar{u}_{1 H}+q_{22}\left(u_{1 T} \bar{v}_{1 H}+v_{1 T} \bar{u}_{1 H}\right)+2 q_{23} v_{1 T} \bar{v}_{1 H}
\end{array}\right),
$$

$$
\mathbf{Q}_{2,5}=\left(\begin{array}{l}
q_{11} u_{1 T}^{2}+q_{12} u_{1 T} v_{1 T}+q_{13} v_{1 T}^{2} \\
q_{21} u_{1 T}^{2}+q_{22} u_{1 T} v_{1 T}+q_{23} v_{1 T}^{2}
\end{array}\right),
$$

$$
\mathbf{Q}_{2,6}=\left(\begin{array}{l}
q_{11} u_{1 H}^{2}+q_{12} u_{1 H} v_{1 H}+q_{13} v_{1 H}^{2} \\
q_{21} u_{1 H}^{2}+q_{22} u_{1 H} v_{1 H}+q_{23} v_{1 H}^{2}
\end{array}\right),
$$

and

$$
\begin{gathered}
z_{2,1}=\left|z_{T}\right|^{2}, \quad z_{2,2}=\left|z_{H}\right|^{2}, \quad z_{2,3}=z_{T} z_{H} e^{i q_{C} x+i \omega_{H} t}, \quad(\mathrm{~A} 7 \mathrm{a}) \\
z_{2,4}=z_{T} \bar{z}_{H} e^{i q_{C} x-i \omega_{H} t}, \quad z_{2,5}=z_{T}^{2} e^{2 i q_{C} x}, \quad z_{2,6}=z_{H}^{2} e^{2 i \omega_{H} t} .
\end{gathered}
$$

Then make the ansatz

$$
\mathbf{u}_{2}=\sum_{j=1}^{2} \mathbf{u}_{2, j} z_{2, j}+\sum_{j=3}^{6}\left(\mathbf{u}_{2, j} z_{2, j}+\text { c.c. }\right) .
$$

By substitution of Eq. (A8) into Eq. (A4), the $\mathbf{u}_{2, j}$ satisfy

$$
\begin{aligned}
& \mathbf{u}_{2,1}=-\frac{1}{\omega_{H}^{2}}\left(\begin{array}{cc}
d & -b \\
-c & a
\end{array}\right) \mathbf{Q}_{2,1}, \\
& \mathbf{u}_{2,2}=-\frac{1}{\omega_{H}^{2}}\left(\begin{array}{cc}
d & -b \\
-c & a
\end{array}\right) \mathbf{Q}_{2,2},
\end{aligned}
$$

$$
\mathbf{u}_{2,5}=-\frac{1}{9 K q_{c}^{4}}\left(\begin{array}{cc}
d-4 K q_{c}^{2} & -b \\
-c & a-4 q_{c}^{2}
\end{array}\right) \mathbf{Q}_{2,5},
$$


The $\mathcal{O}\left(\epsilon^{3}\right)$ problem is

$$
\begin{gathered}
\partial_{t} \mathbf{u}_{3}-\mathbf{L}_{0} \mathbf{u}_{3}=-\partial_{t_{2}} \mathbf{u}_{1}+\mathbf{L}_{2} \mathbf{u}_{1}+\mathbf{N}_{3}\left(\mathbf{u}_{1}, \mathbf{u}_{2}\right)+\mathbf{f} \\
\mathbf{L}_{2}=\left(\begin{array}{lc}
a_{2} & b_{2} \\
c_{2} & d_{2}+K_{2} \nabla^{2}
\end{array}\right), \quad \mathbf{f}=\left(f_{1}, f_{2}\right)^{T}, \quad(\mathrm{~A} 1 \\
\mathbf{N}_{3}\left(\mathbf{u}_{1}, \mathbf{u}_{2}\right)= \\
+\left(\begin{array}{l}
\frac{\partial F_{2}\left(u_{1}, v_{1}\right)}{\partial u_{1}} \\
\frac{\partial G_{2}\left(u_{1}, v_{1}\right)}{\partial u_{1}}
\end{array}\right) u_{2}+\left(\begin{array}{l}
\frac{\partial F_{2}\left(u_{1}, v_{1}\right)}{\partial v_{1}} \\
\frac{\partial G_{2}\left(u_{1}, v_{1}\right)}{\partial v_{1}}
\end{array}\right) v_{2} \\
+\left(\begin{array}{l}
F_{3}\left(u_{1}, v_{1}\right) \\
G_{3}\left(u_{1}, v_{1}\right)
\end{array}\right)
\end{gathered}
$$

There are terms in Eq. (A10) with Fourier dependence $e^{i q x}$ and $e^{i \omega_{H} t}$ for which the operator $\partial_{t}-\mathbf{L}_{0}$ is not invertible. In order to eliminate the secular terms, use the Fredholm alternative theorem. The critical left eigenvectors are $\widetilde{\mathbf{u}}_{1 T} e^{-i q_{c} x}$ and $\widetilde{\mathbf{u}}_{1 H} e^{-i \omega_{H} t}$ where

$$
\tilde{\mathbf{u}}_{1 T}=\left(c_{c},-a_{c}+q_{c}^{2}\right), \quad \tilde{\mathbf{u}}_{1 H}=\left(c_{c},-a_{c}+i \omega_{H}\right) .
$$

Take an inner product of Eq. (A10a) with each in order to obtain the two amplitude equations

$$
\begin{gathered}
\alpha_{T} \frac{d z_{T}}{d t_{2}}=\lambda_{T} z_{T}+g_{1 T}\left|z_{T}\right|^{2} z_{T}+g_{2 T}\left|z_{H}\right|^{2} z_{T}, \\
\alpha_{H} \frac{d z_{H}}{d t_{2}}=F+\lambda_{H} z_{H}+g_{1 H}\left|z_{H}\right|^{2} z_{H}+g_{2 H}\left|z_{T}\right|^{2} z_{H} .
\end{gathered}
$$

The coefficients are given by

$$
\alpha_{T}=\widetilde{\mathbf{u}}_{1 T} \mathbf{u}_{1 T},
$$

$$
\lambda_{T}=\tilde{\mathbf{u}}_{1 T} \mathbf{L}_{2} \mathbf{u}_{1 T}
$$

$$
\begin{aligned}
g_{1 T}= & \tilde{\mathbf{u}}_{1 T}\left[\partial \mathbf{N}_{2}\left(u_{1 T}, v_{1 T}\right) \mathbf{u}_{2,1}+\partial \mathbf{N}_{2}\left(\bar{u}_{1 T}, \bar{v}_{1 T}\right) \mathbf{u}_{2,5}\right. \\
& \left.+3 \beta_{1}\left(u_{1 T}, v_{1 T}\right)\right],
\end{aligned}
$$

$$
g_{2 T}=\widetilde{\mathbf{u}}_{1 T}\left[\partial \mathbf{N}_{2}\left(u_{1 T}, v_{1 T}\right) \mathbf{u}_{2,2}+\partial \mathbf{N}_{2}\left(u_{1 H}, v_{1 H}\right) \mathbf{u}_{2,4}\right.
$$$$
\left.+\partial \mathbf{N}_{2}\left(\bar{u}_{1 H}, \bar{v}_{1 H}\right) \mathbf{u}_{2,3}+\beta_{2}\left(u_{1 H}, v_{1 H}\right) \mathbf{u}_{1 T}\right],
$$$$
\alpha_{H}=\widetilde{\mathbf{u}}_{1 H} \mathbf{u}_{1 H},
$$$$
F=\frac{\omega_{H}}{2 \pi} \int_{0}^{2 \pi / \omega_{H}} \tilde{\mathbf{u}}_{1 H} \mathbf{f} d t
$$

$$
\lambda_{H}=\widetilde{\mathbf{u}}_{1 H} \mathbf{L}_{2} \mathbf{u}_{1 H},
$$

$$
g_{1 H}=\tilde{\mathbf{u}}_{1 H}\left[\partial \mathbf{N}_{2}\left(u_{1 H}, v_{1 H}\right) \mathbf{u}_{2,2}+\partial \mathbf{N}_{2}\left(\bar{u}_{1 H}, \bar{v}_{1 H}\right) \mathbf{u}_{2,6}\right.
$$$$
\left.+3 \beta_{1}\left(u_{1 H}, v_{1 H}\right)\right] \text {, }
$$

$$
\begin{aligned}
g_{2 H}= & \tilde{\mathbf{u}}_{1 H}\left[\partial \mathbf{N}_{2}\left(u_{1 H}, v_{1 H}\right) \mathbf{u}_{2,1}+\partial \mathbf{N}_{2}\left(u_{1 T}, v_{1 T}\right) \overline{\mathbf{u}}_{2,4}\right. \\
& \left.+\partial \mathbf{N}_{2}\left(\bar{u}_{1 T}, \bar{v}_{1 T}\right) \mathbf{u}_{2,3}+\beta_{2}\left(u_{1 T}, v_{1 T}\right) \mathbf{u}_{1 H}\right],
\end{aligned}
$$

where

$$
\partial \mathbf{N}_{2}(u, v)=\left(\begin{array}{ll}
\frac{\partial F_{2}(u, v)}{\partial u} & \frac{\partial F_{2}(u, v)}{\partial v} \\
\frac{\partial G_{2}(u, v)}{\partial u} & \frac{\partial F_{2}(u, v)}{\partial v}
\end{array}\right)
$$

$$
\beta_{1}(u, v)=\left(\begin{array}{l}
u^{2}\left(c_{11} \bar{u}+c_{12} \bar{v}\right)+v^{2}\left(c_{13} \bar{u}+c_{14} \bar{v}\right) \\
u^{2}\left(c_{21} \bar{u}+c_{22} \bar{v}\right)+v^{2}\left(c_{23} \bar{u}+c_{24} \bar{v}\right)
\end{array}\right),
$$

Rescaling Eq. (A12) leads to normal form (2) that we study.

[1] A. M. Turing, Philos. Trans. R. Soc. London, Ser. B 237, 37 (1952).

[2] C. A. Klausmeier, Science 284, 1826 (1999).

[3] Y. J. Li, J. Oslonovitch, N. Mazouz, F. Plenge, K. Krischer, and G. Ertl, Science 291, 2395 (2001).

[4] T. Nozakura and S. Ikeuchi, Astrophys. J. 279, 40 (1984).

[5] V. Castets, E. Dulos, J. Boissonade, and P. DeKepper, Phys. Rev. Lett. 64, 2953 (1990).

[6] Q. Ouyang and H. L. Swinney, Nature (London) 352, 610 (1991).
[7] I. Lengyel, S. Kadar, and I. Epstein, Science 259, 493 (1993).

[8] G. H. Gunaratne, Q. Ouyang, and H. L. Swinney, Phys. Rev. E 50, 2802 (1994).

[9] I. Berenstein, M. Dolnik, L. Yang, A. M. Zhabotinsky, and I. R. Epstein, Phys. Rev. E 70, 046219 (2004).

[10] M. Baurmann, T. Gross, and U. Feudel, J. Theor. Biol. 245, 220 (2007).

[11] M. L. Steyn-Ross, D. A. Steyn-Ross, M. T. Wilson, and J. W. Sleigh, in Advances in Cognitive Neurodynamics, edited by R. Wang, F. Gu, and E. Shen (Humana Press Inc., Totowa, NJ, 
2008), pp. 225-230.

[12] G. Heidemann, M. Bode, and H. Purwins, Phys. Lett. A 177, 225 (1993).

[13] P. V. Paulau, I. V. Babushkin, and N. A. Loiko, Phys. Rev. E 70, 046222 (2004).

[14] M. Meixner, A. De Wit, S. Bose, and E. Schöll, Phys. Rev. E 55, 6690 (1997).

[15] W. Just, M. Bose, S. Bose, H. Engel, and E. Schöll, Phys. Rev. E 64, 026219 (2001).

[16] J. J. Perraud, A. De Wit, E. Dulos, P. DeKepper, G. Dewel, and P. Borckmans, Phys. Rev. Lett. 71, 1272 (1993).

[17] D. G. Miguez, S. Alonso, A. P. Munuzuri, and F. Sagues, Phys. Rev. Lett. 97, 178301 (2006).

[18] S. Alonso, D. G. Miguez, and F. Sagues, EPL 81, 30006 (2008).

[19] S. Alonso and F. Sagues, Phys. Rev. E 80, 035203(R) (2009).

[20] V. K. Vanag and I. R. Epstein, Phys. Rev. Lett. 92, 128301 (2004).

[21] V. K. Vanag and I. R. Epstein, J. Chem. Phys. 131, 104512 (2009).

[22] R. McIlwaine, V. K. Vanag, and I. R. Epstein, Phys. Chem. Chem. Phys. 11, 1581 (2009).

[23] H. Kidachi, Prog. Theor. Phys. 63, 1152 (1980).

[24] A. Rovinsky and M. Menzinger, Phys. Rev. A 46, 6315 (1992).

[25] A. De Wit, D. Lima, G. Dewel, and P. Borckmans, Phys. Rev. E 54, 261 (1996).

[26] I. Lengyel and I. Epstein, Science 251, 650 (1991).

[27] I. Prigogine and R. Lefever, J. Chem. Phys. 48, 1695 (1968).

[28] A. P. Muñuzuri, M. Dolnik, A. M. Zhabotinsky, and I. R. Epstein, J. Am. Chem. Soc. 121, 8065 (1999).

[29] D. G. Míguez, E. M. Nicola, A. P. Muñuzuri, J. Casademunt, F.
Sagués, and L. Kramer, Phys. Rev. Lett. 93, 048303 (2004).

[30] I. Berenstein and A. P. Muñuzuri, Phys. Rev. E 74, 036202 (2006).

[31] S. Rüdiger, D. G. Míguez, A. P. Munuzuri, F. Sagués, and J. Casademunt, Phys. Rev. Lett. 90, 128301 (2003).

[32] D. G. Míguez, V. Pérez-Villar, and A. P. Muñuzuri, Phys. Rev. E 71, 066217 (2005).

[33] A. K. Horváth, M. Dolnik, A. P. Muñuzuri, A. M. Zhabotinsky, and I. R. Epstein, Phys. Rev. Lett. 83, 2950 (1999).

[34] M. Dolnik, A. M. Zhabotinsky, and I. R. Epstein, Phys. Rev. E 63, 026101 (2001).

[35] A. Yochelis, C. Elphick, A. Hagberg, and E. Meron, Europhys. Lett. 69, 170 (2005).

[36] M. Golubitsky, I. Stewart, and D. G. Schaeffer, in Singularities and Groups in Bifurcation Theory, Applied Mathematical Sciences Series No. 69 (Springer-Verlag, New York, 1988), Vol. II.

[37] J. Porter, C. M. Topaz, and M. Silber, Phys. Rev. Lett. 93, 034502 (2004).

[38] C. M. Topaz, J. Porter, and M. Silber, Phys. Rev. E 70, 066206 (2004).

[39] V. M. Eguíluz, M. Ospeck, Y. Choe, A. J. Hudspeth, and M. O. Magnasco, Phys. Rev. Lett. 84, 5232 (2000).

[40] A. Kern and R. Stoop, Phys. Rev. Lett. 91, 128101 (2003).

[41] M. Golubitsky, L. Shiau, C. Postlethwaite, and Y. Zhang, in Coherent Behavior in Neuronal Networks, Spinger Series in Computational Neuroscience, edited by K. Josic, J. Rubin, M. Matías, and R. Romo (Springer, New York, 2009), Vol. 3.

[42] S. L. Judd and M. Silber, Physica D 136, 45 (2000).

[43] L. Yang, A. M. Zhabotinsky, and I. R. Epstein, Phys. Rev. Lett. 92, 198303 (2004). 